\title{
ON THE IDENTIFICATION OF THE COSTS OF SIMULTANEOUS SEARCH
}

\author{
José Luis Moraga-González \\ Zsolt Sándor \\ Matthijs R. Wildenbeest
}


The Public-Private Sector Research Center is a Research Center based at IESE Business School. Its mission is to develop research that analyses the relationships between the private and public sectors primarily in the following areas: regulation and competition, innovation, regional economy and industrial politics and health economics.

Research results are disseminated through publications, conferences and colloquia. These activities are aimed to foster cooperation between the private sector and public administrations, as well as the exchange of ideas and initiatives.

The sponsors of the SP-SP Center are the following:

- Accenture

- Ajuntament de Barcelona

- Departament d' Economia i Coneixement de la Generalitat de Catalunya

- Departament d'Empresa i Ocupació de la Generalitat de Catalunya

- Diputació de Barcelona

- Endesa

- FOBSIC

- Fundació AGBAR

- Institut Català de les Indústries Culturals

- Mediapro

- Sanofi Aventis

- ATM, FGC y TMB

The contents of this publication reflect the conclusions and findings of the individual authors, and not the opinions of the Center's sponsors. 


\title{
On the IDENTIFICATION OF THE Costs OF Simultaneous SEARCH*
}

\author{
José L. Moraga-González ${ }^{\dagger} \quad$ Zsolt Sándor ${ }^{\ddagger} \quad$ Matthijs R. Wildenbeest ${ }^{\S}$
}

Revised: May 2011

\begin{abstract}
This paper studies the identification of the costs of simultaneous search in portfolio problems (Chade and Smith, 2006). We show that market shares data from a single market do not provide sufficient information to identify the search cost distribution in any interval, even if utility distributions are known to the econometrician. We then show that by pooling data from markets where the alternatives that similar decision makers confront vary, the search cost distribution and the utility parameters of the logit demand model can be identified.
\end{abstract}

Keywords: search costs, portfolio choice, non-parametric identification, logit demand JEL Classification: C14, D83, J64

${ }^{*}$ Financial support from Marie Curie Excellence Grant MEXT-CT-2006-042471 is gratefully acknowledged. ${ }^{\dagger}$ ICREA, IESE Business School and University of Groningen, E-mail: jose.1.moraga@gmail.com.

${ }^{\ddagger}$ University of Groningen, E-mail: z. sandor@rug.nl.

${ }^{\S}$ Kelley School of Business, Indiana University, E-mail: mwildenb@indiana.edu. 


\section{Introduction}

The estimation of the costs consumers incur in markets when they search for satisfactory products constitutes a new and important area of empirical research. ${ }^{1}$ The econometrician typically observes the market shares and the characteristics of the different alternatives, but not necessarily the subset of products searched by individual consumers. In markets where the costs of search are significant, variation in the market shares of the distinct alternatives is not just due to variation in their characteristics but also due to variation in the subsets of products different decision-makers choose to search. Therefore, the question is whether the costs of search can be identified with this type of data.

This paper studies the nonparametric identification of the costs of simultaneous search in portfolio problems (Chade and Smith, 2005, 2006). The class of portfolio problems we study embeds a number of important decision problems in economics. In these problems a decision-maker must simultaneously choose among a set of ranked stochastic options; each choice is costly and only the best realized option is finally exercised. This problem arises for example when students apply for colleges (Gale and Shapley, 1962; Kelso and Crawford, 1982; Roth and Sotomayor, 1989), when consumers search for differentiated products (Stigler, 1961; Wolinsky, 1986; Anderson and Renault, 1999), or when workers search for employment (Burdett et al., 2001; Albrecht et al., 2006; Kircher, 2009).

In all these papers, an important issue is the study of the extent to which the costs of search drive a wedge between the market outcome and the social optimum and how it can be corrected. The analysis of counterfactuals requires knowledge of the density of search costs. Therefore, assessments of public policy measures aimed at aligning the market and the social outcomes (minimum wage policy, information disclosure policy, merger policy, etc.), or estimating the social value of new alternatives requires the development of methods to identify and estimate the costs of simultaneous search. In this paper we study whether the restrictions imposed by economic theory allow for the identification of search costs non-parametrically.

We consider markets where the costs of search vary across the decision-makers. In this type of markets, we study whether the econometrician can identify the search cost distribution nonparametrically using market shares data and knowing the utility distributions a large number of options provide to the decision-makers. We first show that such data from a single market do

\footnotetext{
${ }^{1}$ See e.g., Mehta, Rajiv, and Srinivasan (2003), Hortaçsu and Syverson (2004), Hong and Shum (2006), Kim, Bronnenberg and Albuquerque (2009), Honka (2010), Koulayev (2010), Moraga-González, Sándor, and Wildenbeest (2010) and Seiler (2010).
} 
not provide sufficient information to identify the costs of simultaneous search in any reasonable interval. The problem originates from the fact that the sequence of critical search costs that can be identified from the data is convergent, so the set of search cost values the econometrician can identify is not dense in the support of the search cost distribution.

The paper then proceeds by studying whether the use of aggregate data from multiple markets suffices to identify the search cost distribution. We propose to pool data from markets that differ in the alternatives similar decision-makers confront. We show that these data allow for the joint identification of the search cost distribution and the utility parameters in the logit demand model. The reason why this type of data helps is that it generates a distinctive set of search cost values in every market for which the econometrician can retrieve the density of search costs. By pooling market share data from many markets one forces the search cost distribution to be uniquely determined for a much larger set of points. Gathering the appropriate data is relatively easy for the econometrician. For example, in the college problem, one can take data from different towns, with typically distinct numbers of colleges, different application success rates, etc. In the case of consumer search for differentiated products, one can pool data from markets where different product qualities are available.

The remainder of the paper is organized as follows. We start by describing the class of simultaneous search problems we study in Section 2. The identification results use the market share equations and these are derived in Section 3. Two leading examples studied in detail by Chade and Smith $(2005,2006)$ are discussed in Section 4. Our identification results are given in Section 5. Section 6 concludes. The longer proofs are relegated to the Appendix.

\section{The model}

We study the identification of the costs of search in a general class of simultaneous search problems. In this class of problems, a decision-maker must make a simultaneous choice among (ranked) stochastic options; each choice is costly and only the best realized alternative is finally consumed. Chade and Smith $(2005,2006)$ have recently studied these problems and we build on their model. ${ }^{2}$

\footnotetext{
${ }^{2}$ Chade and Smith $(2005,2006)$ study the solution to the simultaneous search problem. They prove that a greedy algorithm finds the solution in two sub-classes of problems: problems with downward recursive (DR) payoff functions, and non-DR problems with prize distributions ordered by a second-order stochastic dominance (SOSD) condition. We instead focus on the identification of the costs of simultaneous search based on market data. Our identification results are for more general payoff structures which need not satisfy DR or SOSD. We nevertheless discuss the DR and SOSD cases in separate subsections.
} 
We assume that in a market there is a continuum of heterogeneous decision-makers who can choose to consume prizes/options from a set $\mathcal{N}$ containing $N$ options. Each option $i$ gives a payoff $u_{i}$, where $u_{i}$ is a random variable with probability distribution $\Psi_{i}$ with support $\Phi_{i} \equiv\left[\underline{u}_{i}, \bar{u}_{i}\right] \subset \Re$, $i=1,2, \ldots, N$. We assume that the random variables $u_{1}, u_{2}, \ldots, u_{N}$ are independent. Let the interval $[0, \bar{u}]$ contain the union of all the options' supports $\left[\underline{u}_{i}, \bar{u}_{i}\right], i=1,2, \ldots, N$. The distribution $\Psi_{i}$ may be discrete or continuous and the support $\Phi_{i}$ may include zero so that some options may fail to give a positive payoff with strictly positive probability. Let us denote by $\mathcal{N}_{F}$ the set of options that can fail with strictly positive probability $\left(\mathcal{N}=\mathcal{N}_{F} \cup \overline{\mathcal{N}}_{F}\right)$.

Assumption FD (free disposal). The set of alternatives $\mathcal{N}$ does not contain strictly dominated options.

The role of this assumption is to exclude options for which market shares are equal to zero. Suppose there exists an option $\ell$ whose payoff is always higher than that of some other option $k$, that is, $\bar{u}_{k} \leq \underline{u}_{\ell}$. Suppose also that option $\ell$ is a "sure" option in the sense that it always yields a positive payoff. In that case, $\ell$ strictly dominates $k$ and $k$ can be discarded from the set of alternatives $\mathcal{N}$. An implication of Assumption FD is that when all options can fail with strictly positive probability, then there does not exist any option that can be ex-ante discarded. Moreover, under Assumption FD, $\cap_{i \in \overline{\mathcal{N}_{F}}} \Phi_{i}$ must be a non-empty interval.

Each decision-maker is characterized by her cost of searching an option. Let $c$ be the cost of search of a decision-maker. If the decision-maker searches the subset of options $\mathcal{S}$, her total cost is $c|\mathcal{S}|$, where $|\mathcal{S}|$ denotes the cardinality of the subset $\mathcal{S}$. Assume $c$ is drawn independently from a common atomless distribution $H(c)$ with support $\Omega=[0, \bar{c}]$; let $h(c)$ denote the corresponding density. It will be convenient to assume $\bar{c}$ is sufficiently large, which ensures that there always exist decision-makers who do not search at all. ${ }^{3}$

Every decision-maker ultimately consumes one single option (like in discrete choice models). For a given set of options $\mathcal{S} \subseteq \mathcal{N}$, let $f(\mathcal{S})$ denote the expected (gross-of-cost) payoff:

$$
f(\mathcal{S}) \equiv E\left[\max \left\{u_{i}: i \in \mathcal{S}\right\}\right]=\int_{0}^{\bar{u}}\left(1-\prod_{i \in \mathcal{S}} \Psi_{i}(u)\right) d u .
$$

Note that $f$ is a non-decreasing and bounded function. We adopt the normalization $f(\varnothing)=0$. The problem of a decision-maker with cost of searching options equal to $c$ is to choose a (sub-)set of options $\mathcal{S} \subseteq \mathcal{N}$ to maximize her expected payoff:

$$
\max _{\mathcal{S} \subseteq \mathcal{N}}\{f(\mathcal{S})-c|\mathcal{S}|\}
$$

\footnotetext{
${ }^{3}$ This assumption can easily be relaxed (see Section 4) and only requires some additional notation.
} 


\section{Market shares}

The problem of identification consists of studying whether the information provided by the market data and the market share equations is sufficient to determine the primitives of the model. We study next the derivation of the market share equations.

A solution to the problem in (2) for decision-maker with search $\operatorname{cost} c$ is a set of options $\mathcal{S}^{*}(c)$. Given this decision-maker chooses to search the set $\mathcal{S}^{*}(c)$, she will consume the best of the options in $\mathcal{S}^{*}(c)$. From the perspective of the entire market, when we vary $c$ the optimal choice set changes. Therefore, to compute the market share of an option $j$, we need to consider all the possible choice sets that contain a given option $j$. Let $\mathcal{S}_{j}$ denote the set of choice sets that include option $j$. Then, aggregating the individual decision-maker choices gives the market-shares of the different alternatives, which we denote by $q_{j}$ :

$$
q_{j}=\sum_{\mathcal{S} \in \mathcal{S}_{j}}\left[\int I(c \text { chooses } \mathcal{S}) d H(c)\right] P_{j \mid \mathcal{S}}
$$

where $I(c$ chooses $\mathcal{S})$ is the indicator that decision-maker $c$ chooses the set of options $\mathcal{S}$ and $P_{j \mid \mathcal{S}}$ is the probability option $j$ turns out to be the best option in the set $\mathcal{S}$.

We next elaborate on these market shares. The solution to problem (2) can be understood as follows. Let $\Sigma_{1}=\arg \max \{f(\{i\}): i \in \mathcal{N}\}$ be the best singleton option, that is, the single alternative that yields the highest payoff to the decision-maker among all possible single options. Likewise, let $\Sigma_{2}=\arg \max \{f(\{i, j\}): i, j \in \mathcal{N}\}$ be the best pair of alternatives, that is, the pair of options that yields the highest payoff to the decision-maker among all possible pairs. More generally, let $\Sigma_{k}=\arg \max \left\{f\left(\left\{i_{1}, i_{2}, \ldots, i_{k}\right\}\right): i_{1}, i_{2}, \ldots, i_{k} \in \mathcal{N}\right\}$ be the $k$-option tuple that gives the highest payoff to the decision-maker. Let $\Sigma_{0}=\varnothing$.

Given the above definition of $\Sigma_{k}$, the optimal choice set for a decision-maker with search cost $c, \mathcal{S}^{*}(c)$, is then equal to $\Sigma_{k^{*}(c)}$ where ${ }^{4}$

$$
k^{*}(c)=\arg \max _{k \in\{0,1, \ldots, N\}}\left\{f\left(\Sigma_{k}\right)-c k\right\} .
$$

Since we have a continuum of decision-makers, we now argue this solution defines a partition of the set of decision-makers into fractions $\mu_{k} \geq 0$ of them choosing to search $k$ options, with $\sum_{k=0}^{N} \mu_{k}=1$.

\footnotetext{
${ }^{4}$ When $N$ is large, finding $k^{*}(c)$ is challenging from a computational point of view because there are $2^{N}$ choice sets to be evaluated. Chade and Smith $(2005,2006)$ provide an algorithm, namely the Marginal Improvement Algorithm (MIA), that identifies the optimal solution for two classes of problems. The first class is one where prizes are binary $\left(\Phi_{i}=\left\{0, u_{i}\right\}\right)$ and the payoff function $f$ satisfies downward recursivity (DR). The second class is one where the prize distributions $\Psi_{i}$ are ordered by a second-order stochastic dominance (SOSD) condition (see Section 4).
} 
The fraction of decision-makers choosing to search $k$ alternatives is

$$
\mu_{k}=\int_{\substack{f\left(\Sigma_{k}\right)-k c \geq f\left(\Sigma_{\ell}\right)-\ell c \\ \ell=0,1,2, \ldots, N}} d H(c), k=0,1,2, \ldots, N
$$

which can be rewritten as

$$
\mu_{k}=I\left(\bar{c}_{k}>\underline{c}_{k}\right)\left[H\left(\bar{c}_{k}\right)-H\left(\underline{c}_{k}\right)\right], k=0,1,2, \ldots, N
$$

where

$$
\begin{aligned}
\bar{c}_{k} & =\min _{\ell<k}\left\{\frac{f\left(\Sigma_{k}\right)-f\left(\Sigma_{\ell}\right)}{k-\ell}\right\} \\
\underline{c}_{k} & =\max _{\ell>k}\left\{\frac{f\left(\Sigma_{\ell}\right)-f\left(\Sigma_{k}\right)}{\ell-k}\right\} .
\end{aligned}
$$

Notice that the indicator $I\left(\bar{c}_{k}>\underline{c}_{k}\right)$ can take value zero, which signifies that no decision-maker will choose to search $k$ options (or the set of options $\Sigma_{k}$ ).

Our identification results build on the market share equations to retrieve the search cost distribution at the set of points generated by the partition (5). We shall refer to these points as cutoff points, because they separate the search cost values that correspond to the different numbers of searches. These cutoff points, which are defined below in (8), follow from the critical search cost values given in (6)-(7). We now state a property of the critical search cost values in (6)-(7) that is useful to determine the cutoff points in (8).

Proposition 1 If (i) $\bar{c}_{k} \geq \underline{c}_{k}$, (ii) $\bar{c}_{k+j} \leq \underline{c}_{k+j}$ for $j=1,2, \ldots, h-1$, and (iii) $\bar{c}_{k+h} \geq \underline{c}_{k+h}$ for some $k \geq 0, h \geq 1, k+h<N$, then

$$
\underline{c}_{k}=\bar{c}_{k+h}=\frac{f\left(\Sigma_{k+h}\right)-f\left(\Sigma_{k}\right)}{h} .
$$

The proof of this result is in the Appendix. Building on this Proposition and on the critical search cost values (6) and (7), we can define the search cost cutoffs that separate the decision-makers choosing to search different numbers of options. These are given by the sequence

$$
\begin{aligned}
c_{0} & =\underline{c}_{0}, \\
c_{k} & =\left\{\begin{array}{ll}
\underline{c}_{k}=\bar{c}_{k+1} & \text { if } \bar{c}_{k} \geq \underline{c}_{k} \\
c_{k-1} & \text { otherwise }
\end{array} \text { for } k \geq 1 .\right.
\end{aligned}
$$

Under the conditions of Proposition 1, at the cutoff point $c_{k}$ decision-makers are indifferent between searching the sets $\Sigma_{k}$ and $\Sigma_{k+h}$. This implies that the sequence of cutoffs $\left\{c_{k}\right\}_{k=0}^{N}$ satisfies an important property. 
Proposition 2 The sequence of cutoff points $\left\{c_{k}\right\}_{k=0}^{N}$ defined in (8) is weakly decreasing.

We now provide a couple of examples to illustrate the cutoffs (8) and the fractions of consumers (5). The purpose of the examples is twofold. First, the examples show that the cutoff sequences are indeed decreasing. Second, and more importantly, the examples serve the purpose of showing that all numbers of options will not necessarily be searched by some decision-makers. In fact, in the second example no decision maker finds it optimal to search 2 options. This is important because such cases pose some threats for identification (cf. Proposition 4,ii).

Example 1. Consider a market where there are 3 options in total. For notational simplicity let $f_{\ell} \equiv f\left(\Sigma_{\ell}\right), \ell=1,2,3$. Let $f_{1}=13, f_{2}=18$ and $f_{3}=20$. A decision-maker with search cost $c$ above $\underline{c}_{0} \equiv \max \left\{f_{1}, f_{2} / 2, f_{3} / 3\right\}=13$ will clearly choose to search the empty set. This is the fraction of consumers denoted $\mu_{0}$ in Figure 1. Since $\underline{c}_{1} \equiv \max \left\{f_{2}-f_{1},\left(f_{3}-f_{1}\right) / 2\right\}=f_{2}-f_{1}$, decision-makers with search cost $c \in\left(\underline{c}_{1}, \bar{c}_{1}\right)=\left(\underline{c}_{1}, \underline{c}_{0}\right)=\left(f_{2}-f_{1}, f_{1}\right)$ clearly prefer to search just one option. This is the fraction of consumers denoted $\mu_{1}$ in the graph. Note that $\underline{c}_{2} \equiv f_{3}-f_{2}$. Therefore, decision-makers with search cost $c \in\left(\underline{c}_{2}, \bar{c}_{2}\right)=\left(\underline{c}_{2}, \underline{c}_{1}\right)=\left(f_{3}-f_{2}, f_{2}-f_{1}\right)$ find it optimal to search 2 options. This is the fraction of consumers $\mu_{2}$ in the graph. Finally, decision-makers with search cost $c \in\left(\underline{c}_{3}, \bar{c}_{3}\right)=\left(\underline{c}_{3}, \underline{c}_{2}\right)=\left(0, f_{3}-f_{2}\right)$ search the three options. This fraction of consumers is denoted $\mu_{3}$ in the Figure.

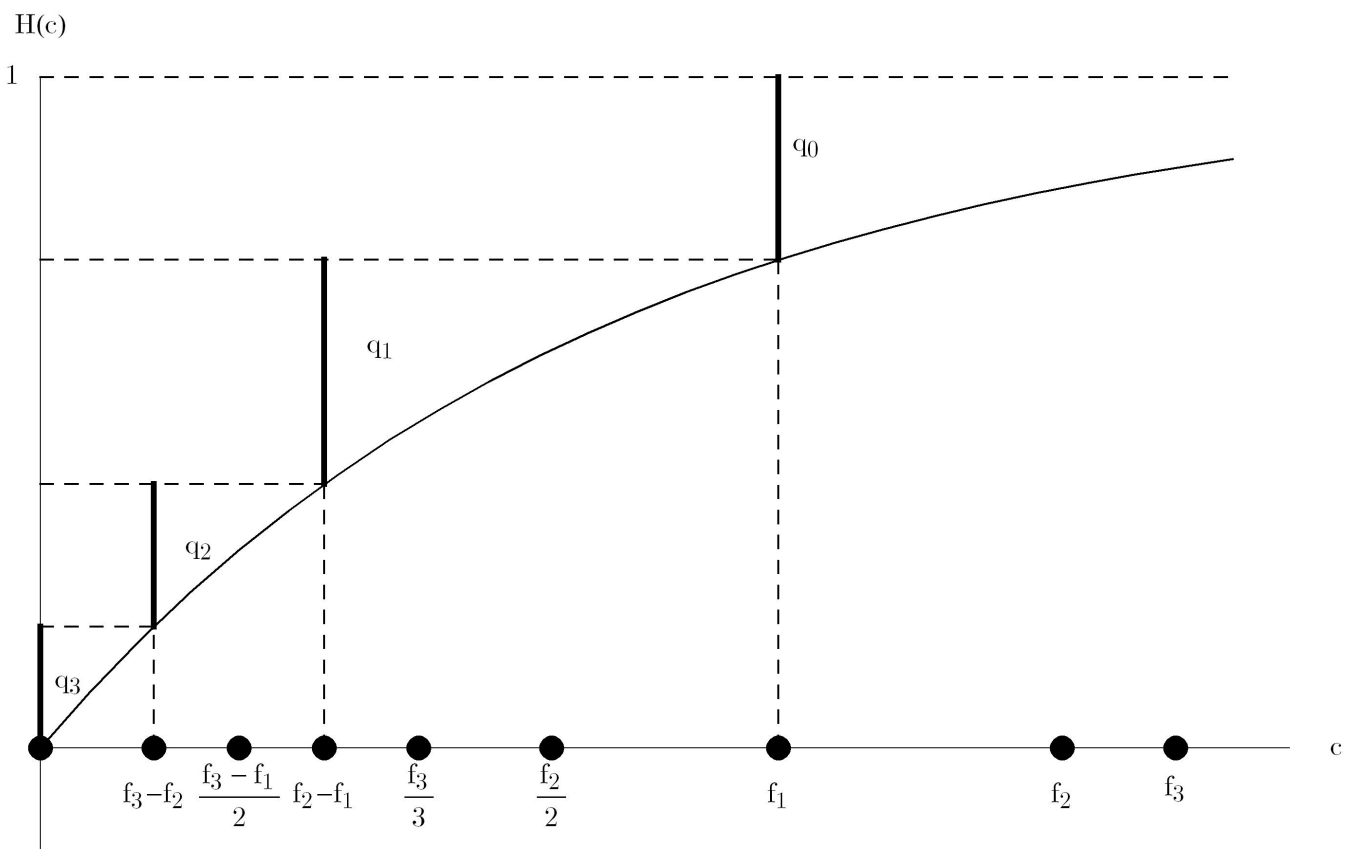

Figure 1: Search decisions in Example 1 
Example 2. This example is exactly identical to the one above except in that we now modify the value of $f_{2}$, which we lower from 18 to 15 . This change is sufficient to render the inequality $\bar{c}_{2} \equiv f_{2}-f_{1}<\underline{c}_{2} \equiv f_{3}-f_{2}$. As it can be seen, in this case the fraction of decision-makers who find it optimal to search two options is equal to zero.

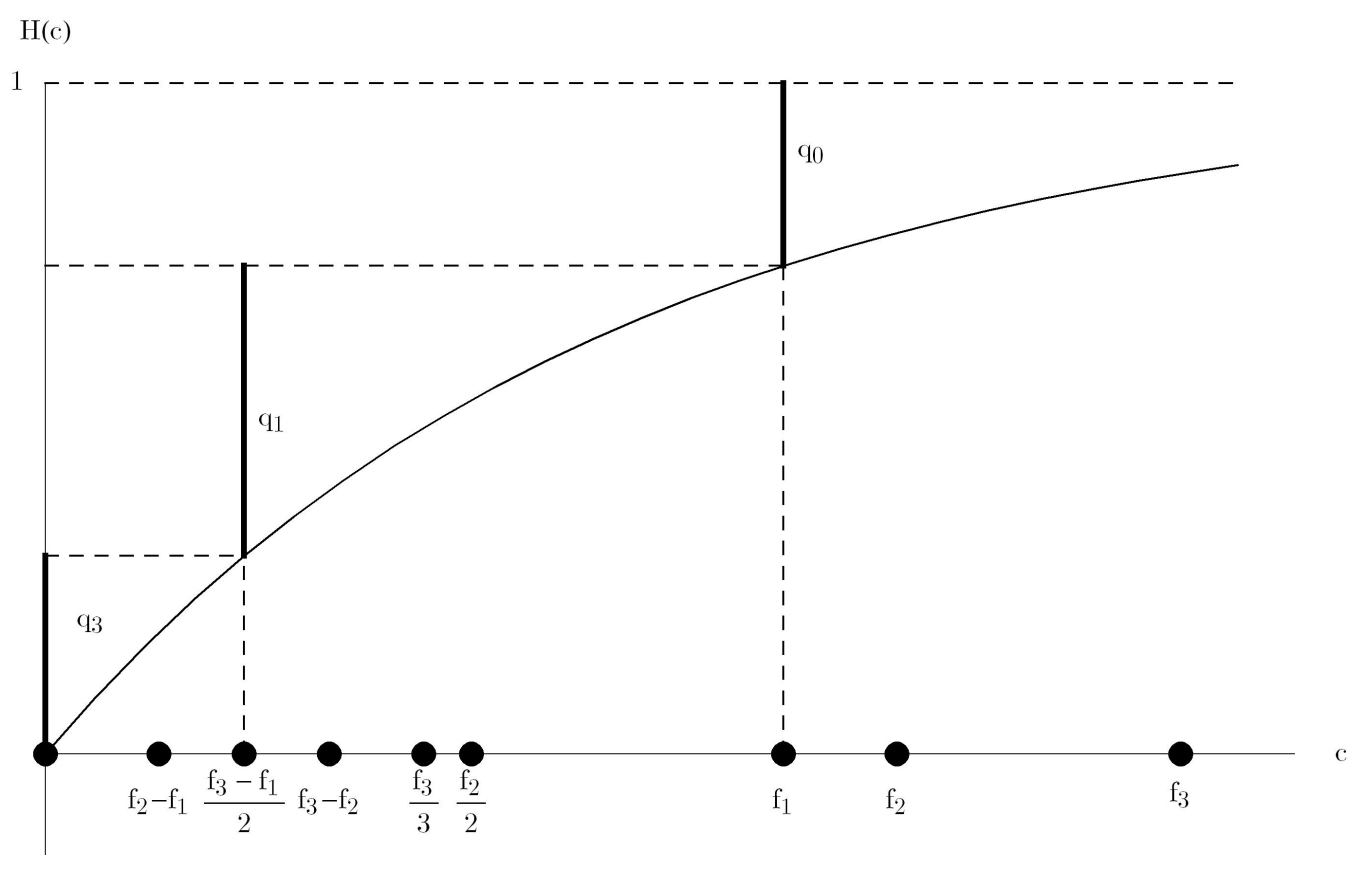

Figure 2: Search decisions in Example 2

We are now ready to compute the market shares of the different alternatives. The system of market shares in (3) can be rewritten as:

$$
\begin{aligned}
q_{0} & =\mu_{0}+\sum_{j=1}^{N} \mu_{j} \sum_{\substack{i_{1}, i_{2} \ldots, i_{j}=1 \\
i_{1}<i_{2}<\ldots<i_{j}}}^{N} \operatorname{Pr}\left[u_{i_{1}}, u_{i_{2}}, \ldots, u_{i_{j}}=0\right] I\left(i_{1}, i_{2}, \ldots, i_{j} \in \Sigma_{j}\right) \\
q_{i} & =\mu_{1} \operatorname{Pr}\left[u_{i}>0\right] I\left(i \in \Sigma_{1}\right) \\
& +\sum_{j=2}^{N} \mu_{j} \sum_{\substack{i_{1}, i_{2} \ldots, i_{j-1}=1 \\
i_{1}, i_{2} \ldots, i_{j-1} \neq i \\
i_{1}<i_{2}<\ldots<i_{j-1}}}^{N} \operatorname{Pr}\left[u_{i}>\max \left\{0, u_{i_{1}}, u_{i_{2}}, \ldots, u_{i_{j-1}}\right\}\right] I\left(i, i_{1}, i_{2}, \ldots, i_{j-1} \in \Sigma_{j}\right)
\end{aligned}
$$

\section{Special cases}

We now present two important cases for which a solution to problem (2) can be found by applying the MIA algorithm of Chade and Smith (2006). These two cases are special in that the payoff 
function $f$ has diminishing returns. As a result, in these two examples all numbers of options will be searched with strictly positive probability, i.e. $\mu_{i}>0$ for all $i$. As shown above, this is not true in general.

\subsection{Binary prizes (The college problem)}

In the college problem ${ }^{5}$ a decision-maker must choose a portfolio of colleges $\mathcal{S} \subseteq \mathcal{N}$ to which to apply for admission. The distributions $\Psi_{i}$ have two-point supports: $\Phi_{i}=\left\{0, u_{i}\right\}$. An option $i$ yields a ex-post payoff $u_{i}$ with probability $\alpha_{i} \in(0,1]$, otherwise zero. The number $\alpha_{i}$ is then the admission chance at college $i$. Moreover, college 1 is ex-post the best, college 2 is ex-post the second best, etc.: $u_{1}>u_{2}>\ldots>u_{N}$. Applying for admission at $\mathcal{S} \subseteq \mathcal{N}$ colleges costs the student $c|\mathcal{S}|$. The expected (gross-of-cost) payoff from applying to the set of colleges $\mathcal{S}$ is

$$
f(\mathcal{S})=\sum_{i=1}^{|\mathcal{S}|} \alpha_{(i)} u_{(i)} \prod_{j=1}^{i-1}\left(1-\alpha_{(j)}\right)
$$

where $(i)$ denotes the $i$-th best ranked college in the set $\mathcal{S}$ and therefore $\prod_{j=1}^{i-1}\left(1-\alpha_{(j)}\right)$ is the probability with which a decision-maker gets rejected by the top-ranked $i-1$ schools in $\mathcal{S}$. Chade and Smith (2006) point out that the payoff function in (10) is downward recursive (DR). That is, for any two sets $U, L$ in $\mathcal{N}$ with $U \sqsupseteq L$ (i.e. where the worst option in $U$ beats the best in $L$ ) we have $f(U+L)=f(U)+\rho(U) f(L)$ where $\rho(U) \equiv \prod_{j \in U}\left(1-\alpha_{j}\right)$ is the chance that all the options in the set $U$ fail.

Chade and Smith (2006) provide an algorithm to find $\mathcal{S}^{*}(c)$. The Marginal Improvement Algorithm (MIA) identifies the solution to this problem via an inductive procedure. In essence, the MIA algorithm works as follows.

Step 1. Add the best single option $\sigma_{1}=\arg \max \left\{\alpha_{i} u_{i}: i \in \mathcal{N}\right\}$ to the optimal choice set if $f\left(\left\{\sigma_{1}\right\}\right) \geq c$, otherwise choose the empty set and stop.

Step 2. Add option $\sigma_{2}=\arg \max \left\{f\left(\left\{\sigma_{1}, i\right\}\right): i \in \mathcal{N}\left\{\sigma_{1}\right\}\right\}$ to the optimal choice set if $f\left(\left\{\sigma_{1}, \sigma_{2}\right\}\right)-f\left(\left\{\sigma_{1}\right\}\right) \geq c$, otherwise stop.

Step $k$. Add option $\sigma_{k}=\arg \max \left\{f\left(\left\{\sigma_{1}, \sigma_{2}, \ldots, \sigma_{k-1}, i\right\}\right): i \in \mathcal{N} \backslash\left\{\sigma_{1}, \sigma_{2}, \ldots, \sigma_{k-1}\right\}\right\}$ to the optimal choice set if $f\left(\left\{\sigma_{1}, \sigma_{2}, \ldots, \sigma_{k}\right\}\right)-f\left(\left\{\sigma_{1}, \sigma_{2}, \ldots, \sigma_{k-1}\right\}\right) \geq c$, otherwise stop (for all $k=$ $3,4, . ., N)$.

\footnotetext{
${ }^{5}$ The college problem is similar to the directed search problem in labor economics studied by Burdett, Shi and Wright (2001), Albrecht et al. (2006) and Kircher (2009).
} 
For our purposes, we are interested in the market outcome this problem generates. Recall that $\mu_{i}$ denotes the probability that a randomly selected decision-maker chooses the set of options $\left\{\sigma_{1}, \sigma_{2}, \ldots, \sigma_{i}\right\}, i=1,2, . ., N$, and $\mu_{0}$ is the probability of choosing the empty set. Since $c$ is distributed according to $H$, these probabilities can readily be computed:

$$
\begin{aligned}
& \mu_{0}=1-H\left(c_{0}\right) \text { and } \\
& \mu_{k}=H\left(c_{k-1}\right)-H\left(c_{k}\right), k=1,2, \ldots, N,
\end{aligned}
$$

where

$$
\begin{aligned}
c_{0} & =f\left(\left\{\sigma_{1}\right\}\right), \\
c_{k} & =f\left(\left\{\sigma_{1}, \sigma_{2}, \ldots, \sigma_{k+1}\right\}\right)-f\left(\left\{\sigma_{1}, \sigma_{2}, \ldots, \sigma_{k}\right\}\right), k=1,2, \ldots, N-1 \\
c_{N} & =0 .
\end{aligned}
$$

The number $c_{k}$ is the corresponding cutoff value of the search cost distribution that makes a decisionmaker indifferent between choosing the best $k$-option-tuple and the best $k+1$-option-tuple. We note that the sequence of cutoff values $\left\{c_{i}\right\}_{i=0}^{N}$ is decreasing because $f$ exhibits diminishing returns (see Lemma 5 in Chade and Smith, 2006); therefore the probabilities $\mu_{i}$ are well-defined and are all strictly positive.

The market shares of the different options can be written as follows:

$$
\begin{aligned}
q_{0} & =\mu_{0}+\sum_{j=1}^{N} \mu_{j} \prod_{\ell=1}^{j}\left(1-\alpha_{\sigma_{\ell}}\right) \\
q_{\sigma_{k}} & =\alpha_{\sigma_{k}} \prod_{\ell=1}^{k-1}\left(1-\alpha_{\sigma_{\ell}}\right) \sum_{j=k}^{N} \mu_{j}, k=1,2, \ldots, N .
\end{aligned}
$$

where $\alpha_{\sigma_{\ell}}$ is the probability with which the application of a decision-maker succeeds at college $\sigma_{\ell}$.

\subsection{Search for differentiated products}

Consider the following model of search for differentiated products, which generalizes Stigler (1961) and Burdett and Judd (1983) to the case of non-identical and non-sure prizes (Chade and Smith, 2005). There are $N$ firms offering differentiated products to a continuum of consumers. A consumer must visit a set $\mathcal{S}$ of shops to learn the utility she derives from the different products available at the shops in $\mathcal{S}$. Once the utility is learnt, the consumer picks the single product that gives her the highest utility. Assume that the product of a shop $i$ gives a payoff $u_{i}$ with probability $\alpha_{i} \in(0,1]$, 
where $u_{i}$ is a random variable with probability distribution $\Psi_{i}$ and support $\left[\underline{u}_{i}, \bar{u}_{i}\right], i=1,2, \ldots, N$. The scalar $\alpha_{i}$ can be interpreted here as the probability with which a shop carries the product, or has it in stock. Assume that the random variables $u_{1}, u_{2}, \ldots, u_{N}$ are independent. Let the interval $[0, \bar{u}]$ contain the union of all the products' supports $\left[\underline{u}_{i}, \bar{u}_{i}\right], i=1,2, \ldots, N$. Define $\nu_{i}=I_{\alpha_{i}} u_{i}$, where $I_{\alpha_{i}}$ is a Bernoulli random variable that takes value 1 with probability $\alpha_{i}$. The distribution of $\nu_{i}$ is $G_{i}=\left(1-\alpha_{i}\right)+\alpha_{i} \Psi_{i}$ and its support is $\Phi_{i} \equiv\{0\} \cup\left[\underline{u}_{i}, \bar{u}_{i}\right]$ when $\alpha_{i}<1$, otherwise $\Phi_{i} \equiv\left[\underline{u}_{i}, \bar{u}_{i}\right]$.

Assumption SOSD. The distributions $G_{i}$ can be ranked according to the quasi-second order stochastic dominance criterion, that is: $\int_{x}^{\bar{u}} G_{i}(u) d u \leq \int_{x}^{\bar{u}} G_{i+1}(u) d u, i=1,2, \ldots, N-1$, for all $x \in[0, \bar{u}]$ with strict inequality at $x=0$.

Given Assumption SOSD, ex-ante the best product is product 1, the second best product is product 2, etc. The expected (gross-of-cost) payoff from visiting the set of shops $\mathcal{S}$ is

$$
f(\mathcal{S}) \equiv \int_{0}^{\bar{u}}\left(1-\prod_{i \in \mathcal{S}} G_{i}(u)\right) d u
$$

Chade and Smith (2005) note that the payoff structure in (14) is not DR.

For our purpose, it is useful to prove that $f$ has the following properties.

Proposition 3 Under Assumption SOSD, ${ }^{6}$ :

(i) $f(\{1,2, \ldots, i\}) \geq f(\{1,2, \ldots, i-1, \ell\})$ for all $\ell \geq i$.

(ii) $f(\{1,2, \ldots, i\})-f(\{1,2, \ldots, i-1\}) \geq f(\{1,2, \ldots, i+1\})-f(\{1,2, \ldots, i\}), i=2, \ldots, N$.

Proof. (i) We need to prove that

$$
\int_{0}^{\bar{u}}\left(1-\prod_{j=1}^{i} G_{j}(u)\right) d u-\int_{0}^{\bar{u}}\left(1-G_{\ell}(u) \prod_{j=1}^{i-1} G_{j}(u)\right) d u=\int_{0}^{\bar{u}} \prod_{j=1}^{i-1} G_{j}(u)\left(G_{\ell}(u)-G_{i}(u)\right) d u \geq 0
$$

for all $\ell \geq i$. Notice that $G_{\ell}(u)-G_{i}(u)$ can be negative for some $u$. Let $\beta(u)=\prod_{j=1}^{i-1} G_{j}(u)$ and $\gamma(u)=G_{\ell}(u)-G_{i}(u)$. We need to show that

$$
\int_{0}^{\bar{u}} \beta(u) \gamma(u) d u \geq 0
$$

Note that $\beta$ is monotonically increasing in $u$, is absolutely continuous on $(0, \bar{u}]$, with $\beta(0)=0$ and $\beta(\bar{u})=1$. Moreover, by the SOSD assumption, $\int_{x}^{\bar{u}} \gamma(u) d u \geq 0$ for all $x \in[0, \bar{u}] .{ }^{7}$ Let

$$
\Gamma(x)=-\int_{x}^{\bar{u}} \gamma(u) d u .
$$

\footnotetext{
${ }^{6}$ In fact, we do not need the strict inequality condition at $x=0$.

${ }^{7}$ We note that $\gamma(u)$ may cross the horizontal axis several times.
} 
Integrating (15) by parts gives

$$
\begin{aligned}
\int_{0}^{\bar{u}} \beta(u) \gamma(u) d u & =\beta(\bar{u}) \Gamma(\bar{u})-\beta(0) \Gamma(0)-\int_{0}^{\bar{u}} \Gamma(u) \frac{d \beta(u)}{d u} d u \\
& =-\prod_{j=1}^{i-1}\left(1-\alpha_{j}\right) \Gamma(0)-\int_{0}^{\bar{u}} \Gamma(u) \frac{d \beta(u)}{d u} d u \geq 0 .
\end{aligned}
$$

so the result follows.

(ii) In this case we need to show that

$$
\begin{aligned}
& \int_{0}^{\bar{u}}\left(1-\prod_{j=1}^{i} G_{j}(u)\right) d u-\int_{0}^{\bar{u}}\left(1-\prod_{j=1}^{i-1} G_{j}(u)\right) d u-\int_{0}^{\bar{u}}\left(1-\prod_{j=1}^{i+1} G_{j}(u)\right) d u+\int_{0}^{\bar{u}}\left(1-\prod_{j=1}^{i} G_{j}(u)\right) d u \\
& =\int_{0}^{\bar{u}}\left(\prod_{j=1}^{i-1} G_{j}(u)-2 \prod_{j=1}^{i} G_{j}(u)+\prod_{j=1}^{i+1} G_{j}(u)\right) d u=\int_{0}^{\bar{u}}\left(\prod_{j=1}^{i-1} G_{j}(u)\left(1-2 G_{i}(u)+G_{i}(u) G_{i+1}(u)\right)\right) d u \geq 0 .
\end{aligned}
$$

We now argue that

$$
\int_{0}^{\bar{u}}\left(\prod_{j=1}^{i-1} G_{j}(u)\left(1-2 G_{i}(u)+G_{i}(u) G_{i+1}(u)\right)\right) d u \geq \int_{0}^{\bar{u}}\left(\prod_{j=1}^{i-1} G_{j}(u)\left(1-G_{i}(u)\right)^{2}\right) d u \geq 0 .
$$

For this, it suffices that

$$
\int_{0}^{\bar{u}} \rho(u) \tau(u) d u \geq 0
$$

where $\rho(u)=\prod_{j=1}^{i} G_{j}(u)$ and $\tau(u)=G_{i+1}(u)-G_{i}(u)$. But this follows from the proof of (i).

We can use this proposition to describe the market outcome this problem generates. The solution to the problem of a consumer with search cost $c$ is a set of options $\left\{1,2, \ldots, i^{*}(c)\right\}$. From Part (i) in the Proposition, we derive the following algorithm to encounter the optimal solution. ${ }^{8}$

Step 1. Add option 1 to the optimal choice set if $f(\{1\}) \geq c$, otherwise choose the empty set and stop.

Step 2. Add option 2 to the optimal choice set if $f(\{1,2\})-f(\{1\}) \geq c$, otherwise stop.

Step $i$. Add option $i$ to the optimal choice set if $f(\{1,2, \ldots, i\})-f(\{1,2, \ldots, i-1\}) \geq c$, otherwise stop (for all $i=3,4, . ., N$ ).

Since $c$ is distributed according to $H$, the probability $\mu_{i}$ that a randomly selected decision-maker chooses to sample the set of options $\{1,2, \ldots, i\}, i=1,2, . ., N$, can readily be computed:

$$
\begin{aligned}
\mu_{0} & =1-H\left(c_{0}\right) \text { and } \\
\mu_{i} & =H\left(c_{i-1}\right)-H\left(c_{i}\right), i=1,2, \ldots, N,
\end{aligned}
$$

\footnotetext{
${ }^{8}$ This algorithm succeeds in $O(N)$ steps.
} 
where

$$
\begin{aligned}
c_{0} & =f(\{1\}), \\
c_{i} & =f(\{1,2, \ldots, i+1\})-f(\{1,2, \ldots, i\}), i=1,2, \ldots, N-1 \\
c_{N} & =0 .
\end{aligned}
$$

The number $c_{i}$ in this case is the cutoff value of the search cost distribution that makes a decisionmaker indifferent between choosing $i$ and $i+1$ options. By Proposition 3, the sequence of cutoff values $\left\{c_{i}\right\}_{i=0}^{N}$ is decreasing and therefore the probabilities $\mu_{i}$ are all strictly positive.

Let $q_{i}$ denote the market share of option $i$. Computing the market share of an option $i$ involves summing over all $\mu$ 's the probability option $i$ turns out to be the most attractive. In general this market share can be written as follows:

$$
\begin{aligned}
q_{0} & =\mu_{0}, \\
q_{1} & =\mu_{1}+\mu_{2} \operatorname{Pr}\left(\nu_{1}>\nu_{2}\right)+\ldots+\mu_{N} \operatorname{Pr}\left(\nu_{1}>\max \left\{\nu_{2}, \nu_{3} \ldots, \nu_{N}\right\}\right), \\
q_{2} & =\mu_{2} \operatorname{Pr}\left(\nu_{2}>\nu_{1}\right)+\ldots+\mu_{N} \operatorname{Pr}\left(\nu_{2}>\max \left\{\nu_{1}, \nu_{3} \ldots, \nu_{N}\right\}\right), \\
& \ldots \\
q_{N} & =\mu_{N} \operatorname{Pr}\left(\nu_{N}>\max \left\{\nu_{1}, \nu_{2}, \ldots, \nu_{N-1}\right\}\right) .
\end{aligned}
$$

\section{Identification of the costs of search}

The econometrics problem consists of retrieving the costs of simultaneous search, i.e., the distribution function $H$, from the market-share equations given in (9). A crucial requirement for consistent estimation is that the search cost distribution is identified. In what follows, we study whether such identification is possible and the necessary data requirements. We start with the case where the econometrician has information about the preferences of the decision-makers. The joint identification of preferences and search costs is postponed to section 5.2.

\subsection{Identification with known preferences}

In this section we treat the case of identification based on data from a single market.

Assumption DTA1. In a given market, the econometrician observes:

1. The distributions $\left\{\Psi_{i}\right\}_{i=1}^{N}$ over the set of prizes $\left\{\Phi_{i}\right\}_{i=1}^{N}$ the options can deliver.

2. The aggregate market shares of the options, denoted $\left\{q_{i}\right\}_{i=0}^{N}$, where $q_{0}$ is the market share of the "outside" option. 
In the light of the special cases discussed in Section 4, Assumption DTA1 requires observing the numbers of students accepted at the different colleges or observing the market shares of the various products in a single market. Assumption 3.1 implies that the researcher knows the utility distributions each option can yield. Admittedly, this is a significant amount of information and therefore this assumption represents a case quite favorable for identification of the search cost distribution. Even in this favorable situation, we shall point to some identification challenges (which of course would remain if the utility distributions were not known to the econometrician). Later in Section 5.2 we discuss the joint identification of search costs and utility distributions.

The econometrician may be able to obtain additional data on the number of searches. Consider then:

Assumption DTA2. The econometrician observes the data in Assumption DTA1 and in addition the distribution of the number of searches in the market.

Our first result studies if the data described above allow for the identification of the search cost distribution at the cutoff values defined in (8).

Proposition 4 (i) Under Assumption DTA1, in the binary-prize model, and in the search-fordifferentiated-products model with SOSD, the search cost cutoff values $\left\{c_{i}\right\}_{i=0}^{N}$ and the corresponding values of the CDF of search cost $\left\{H\left(c_{i}\right)\right\}_{i=0}^{N}$ are identified.

(ii) Under Assumption DTA1, in the general search model, the search cost cutoff values $\left\{c_{i}\right\}_{i=0}^{N}$ and the corresponding values of the CDF of search cost $\left\{H\left(c_{i}\right)\right\}_{i=0}^{N}$ need not be identified. However, under Assumption DTA2, they are identified.

Proof. (i) In the binary prize model, $\left\{\sigma_{k}\right\}_{k=1}^{N}$ can be computed by the MIA algorithm. The interesting case is when the probability the application of a decision-maker succeeds at a college $\alpha_{\sigma_{k}}<1$, for all $k<N .{ }^{9}$ The cutoff values can be computed from the set of equalities (12). Market shares satisfy the system of equations in (13). Note that this system of equations is triangular and it has strictly positive diagonal elements ( $k$-th diagonal element is $\left.\alpha_{\sigma_{k}} \prod_{\ell=1}^{k-1}\left(1-\alpha_{\sigma_{\ell}}\right)>0\right)$. Therefore the system can be solved for $\left\{\mu_{k}\right\}_{k=0}^{N}$. Once the fractions of students who apply to $k$ colleges is obtained, one can iteratively compute the corresponding values of the CDF of the costs of simultaneous search at the cutoffs:

$$
H\left(c_{i}\right)=1-\sum_{i=0}^{k} \mu_{i} \text { where } k=0,1, \ldots, N .
$$

\footnotetext{
${ }^{9}$ Otherwise, if $\alpha_{\sigma_{k}}=1$ for some $k<N$ then from (13) it follows $q_{\sigma_{N}}=0$, so Assumption FD is violated.
} 
In the model of search for differentiated products, the SOSD assumption makes the arguments similar. From the equalities (18) one can compute the cutoff values $\left\{c_{k}^{N}\right\}_{k=1}$. Using the market shares in the system of equations (19) we can compute $\left\{\mu_{k}\right\}_{k=0}^{N}$. Again this system is triangular with $k$-th diagonal element equal to $\operatorname{Pr}\left(\nu_{k}>\max \left\{\nu_{1}, \nu_{2}, \ldots, \nu_{k-1}\right\}\right)>0$. Too see this, denote by $L \subset\{1,2, \ldots, k-1\}$ the subset of options that fail with strictly positive probability. Let $\rho(L)$ be the probability that all options in $L$ fail; by convention $\rho(\varnothing)=1$. Then $\operatorname{Pr}\left(\nu_{k}>\max \left\{0, \nu_{1}, \nu_{2}, \ldots, \nu_{k-1}\right\}\right) \geq \rho(L) \operatorname{Pr}\left(\nu_{k}>\max \left\{0, \max \left\{\nu_{i}: i \in \bar{L}\right\}\right\}\right)$. If option $k$ succeeds with probability 1, then by Assumption SOSD, $\operatorname{Pr}\left(\nu_{k}>\max \left\{\nu_{i}: i \in \bar{L}\right\}\right)>0$. If option $k$ fails with strictly positive probability, by Assumption FD on free disposal of strictly dominated options, it must be the case that the upper bound of the support of option $k, \bar{u}_{k}>\max \left\{\underline{u}_{i}: i \in \bar{L}\right\}$. As a result, $\operatorname{Pr}\left(\nu_{k}>\max \left\{\nu_{i}: i \in \bar{L}\right\}\right)>0$. Once the fractions of consumers sampling $k$ options are known, one can iteratively compute the corresponding values of the CDF of search costs as before.

(ii) In the general model, the system of market shares might not be invertible and so we may be unable to solve for $\left\{\mu_{k}\right\}_{k=0}^{N}$. This precludes identification of the search cost distribution at the cutoffs under Assumption DTA1. To illustrate the problem, let us go back to the case described above in Example 1 where there are three options in total and $f_{1}=13, f_{2}=18$ and $f_{3}=20$, where $f_{\ell} \equiv f\left(\Sigma_{\ell}\right), \ell=1,2,3$. Assume also that $\Sigma_{1}=\{1\}$, and $\Sigma_{2}=\{2,3\}$. Finally, assume that $\Psi_{i}$ can only have an atom at zero. Then, the system of market shares is

$$
\left(\begin{array}{c}
q_{1} \\
q_{2} \\
q_{3}
\end{array}\right)=\left(\begin{array}{ccc}
\operatorname{Pr}\left[u_{1}>0\right] & 0 & \operatorname{Pr}\left[u_{1}>\max \left\{0, u_{2}, u_{3}\right\}\right] \\
0 & \operatorname{Pr}\left[u_{2}>\max \left\{0, u_{3}\right\}\right] & \operatorname{Pr}\left[u_{2}>\max \left\{0, u_{1}, u_{3}\right\}\right] \\
0 & \operatorname{Pr}\left[u_{3}>\max \left\{0, u_{2}\right\}\right] & \operatorname{Pr}\left[u_{3}>\max \left\{0, u_{1}, u_{2}\right\}\right]
\end{array}\right)\left(\begin{array}{c}
\mu_{1} \\
\mu_{2} \\
\mu_{3}
\end{array}\right)
$$

Notice that the matrix of coefficients in this system need not be triangular. In fact, the system is not invertible provided that

$$
\operatorname{Pr}\left[u_{2}>\max \left\{0, u_{3}\right\}\right] \operatorname{Pr}\left[u_{3}>\max \left\{0, u_{1}, u_{2}\right\}\right]=\operatorname{Pr}\left[u_{2}>\max \left\{0, u_{1}, u_{3}\right\}\right] \operatorname{Pr}\left[u_{3}>\max \left\{0, u_{2}\right\}\right] .
$$

Under the data assumption DTA2, the econometrician observes $\left\{\mu_{k}\right\}_{k=0}^{N}$ and then the search cost distribution at the cutoffs is identified.

Proposition 4 gives conditions under which one can identify the sequence of points $\left\{c_{i}, H\left(c_{i}\right)\right\}_{i=0}^{N}$ of the search cost distribution. Clearly, a limited number of options will not suffice to identify a density. The question that arises is whether taking a market with a sufficiently large number of options can allow for the identification of the search cost distribution with enough precision. The next result argues that it is impossible even if the number of options goes to infinity. 
Proposition 5 Under Assumptions DTA1 or DTA2, the search cost distribution is not identified in any interval of interest in either of the models (the binary-prize model, the search-for-differentiatedproducts model with SOSD and the general model), even if $N \rightarrow \infty$.

Proof. From Proposition 2, the sequence of cutoffs is weakly decreasing and therefore convergent. As a result, the set of points outside an arbitrarily small neighborhood around the limit point will necessarily be finite. This implies that the search cost distribution is not identified in any interval of interest.

Proposition 5 shows that using the type of aggregate data described in Assumption DTA1 and DTA2 only allows for identification of the costs of simultaneous search around the limit point of the sequence of cutoffs. This constitutes an important problem because any reasonable study of the effects of public policy measures aimed at improving the market outcome in any of the settings described above (for instance, introducing new college options, increasing school places, challenging a merger between options, etc.) would require the identification of search costs at all quantiles. We now proceed to study the identification of the search cost distribution in richer settings.

\subsection{Joint identification of preferences and search costs}

In the previous section we have pointed out that, even if the econometrician knows the preferences of decision-makers, the search cost distribution cannot be identified using data from a single market. In this section we argue that by pooling data from several markets it is possible to identify the search cost distribution non-parametrically. ${ }^{10}$ Such an identification result would not be very useful from a practical point of view if preferences of decision-makers were not identifiable. Therefore in this Section we focus on the joint identification of preferences and search costs.

We study this question in the widely used random utility framework that leads to logit demands. Therefore, we consider that the payoff of an option $i$ in a certain market is given by

$$
u_{i}=\mathbf{x}_{i} \beta+\varepsilon_{i}
$$

where the vector $\mathbf{x}_{i}$ represents the (observable) characteristics of option $i$, the vector $\beta$ represents the importance of each characteristic in the payoff of a decision-maker and $\varepsilon_{i}$ is a random variable that represents the match between a decision maker and the option $i$. We assume $\varepsilon_{i}$ to be type-I extreme value distributed.

\footnotetext{
${ }^{10}$ This strategy is related to the literature on nonparametric identification of the distribution of random coefficients in discrete choice models. For example, the identification result in Bajari et al. (2010), although based on a different formal argument, also requires pooling market share data from several markets.
} 
We assume that the purpose of searching is to discover the match values $\varepsilon_{i}$ of the alternatives chosen to be searched. Meanwhile, the vectors of characteristics $\mathbf{x}_{i}$ of the various options are assumed to be known at the moment the decision-maker chooses her set of alternatives $\mathcal{S}$ to be searched. Once the options in the choice set $\mathcal{S}$ are evaluated and the decision-maker learns the match values, the decision-maker picks one of the options to consume, or else takes the outside option, whose utility is normalized to zero.

The expected (gross-of-cost) payoff from visiting the set of shops $\mathcal{S}$ is

$$
f(\mathcal{S}) \equiv \int\left(1-\prod_{i \in \mathcal{S}} G_{i}(u)\right) d u=\gamma+\log \left(\sum_{i \in \mathcal{S}} \exp \left[\mathbf{x}_{i} \beta\right]\right)
$$

where $\gamma$ is the Euler constant. Without loss of generality, assume that the best product ex-ante has index 1 , the second best has index 2 , etc., that is, $\mathbf{x}_{1} \beta \geq \mathbf{x}_{2} \beta \geq \ldots \geq \mathbf{x}_{N} \beta$. Then it is easy to see that the payoff function $f$ in (21) satisfies the properties from Proposition 3, that is, (i) $f(\{1,2, \ldots, i\}) \geq f(\{1,2, \ldots, i-1, \ell\})$ for all $\ell \geq i$ and (ii) $f(\{1,2, \ldots, i\})-f(\{1,2, \ldots, i-1\}) \geq$ $f(\{1,2, \ldots, i+1\})-f(\{1,2, \ldots, i\}), i=2, \ldots, N$. Therefore, using (18), the cutoff points are given by

$$
\begin{aligned}
c_{0}= & f(\{1\})-f(\varnothing)=\gamma+\mathbf{x}_{1} \beta, \\
c_{1}= & f(\{1,2\})-f(\{1\})=\log \left(\exp \left[\mathbf{x}_{1} \beta\right]+\exp \left[\mathbf{x}_{2} \beta\right]\right)-\mathbf{x}_{1} \beta, \\
& \vdots \\
c_{N-1}= & \log \left(\sum_{i=1}^{N} \exp \left[\mathbf{x}_{i} \beta\right]\right)-\log \left(\sum_{i=1}^{N-1} \exp \left[\mathbf{x}_{i} \beta\right]\right), \\
c_{N}= & 0 .
\end{aligned}
$$

Note that from (17) and (19) we obtain that $H\left(\gamma+\mathbf{x}_{1} \beta\right)=1-q_{0}$.

Suppose that the econometrician observes the market shares and the characteristics of the options in $M$ different markets. Assume that the vector of characteristics of the best product in market $m$, denoted $\mathbf{x}_{1}^{m}$, is a random draw from a distribution with continuum support such that the random variables $\gamma+\mathbf{x}_{1}^{m} \beta_{0}$ cover the support of $H$, where $\beta_{0}$ is the true parameter vector. Then for given $\beta$, we can identify the search cost distribution $H_{\beta}$ for which $H_{\beta}\left(\gamma+\mathbf{x}_{1}^{m} \beta\right)=1-q_{0}^{m}$, for all $m$, on some support that may depend on $\beta$. In order to show that the we can identify the true $\beta_{0}$, suppose by contradiction that for another parameter, say $\beta_{1}$, we have the same search cost distribution, that is, $H_{\beta_{0}}=H_{\beta_{1}}$. This implies that $\gamma+\mathbf{x}_{1}^{m} \beta_{0}=H_{\beta_{0}}^{-1}\left(1-q_{0}^{m}\right)=H_{\beta_{1}}^{-1}\left(1-q_{0}^{m}\right)=$ $\gamma+\mathbf{x}_{1}^{m} \beta_{1}$ for all $m$, so $X_{1}\left(\beta_{0}-\beta_{1}\right)=0$, where $X_{1}=\left(\left(\mathbf{x}_{1}^{1}\right)^{\prime},\left(\mathbf{x}_{1}^{2}\right)^{\prime}, \ldots,\left(\mathbf{x}_{1}^{M}\right)^{\prime}\right)^{\prime}$. If $M$ is greater than or equal to the dimension of the vector of characteristics, this can only happen if $X_{1}$ does not have full rank, which is a zero-probability event. As a result, we conclude that $\beta_{0}$ is identified and $H_{\beta_{0}}$ identifies the true search cost distribution. To summarize: 
Proposition 6 Consider the simultaneous search model with logit demand described above. Assume the econometrician has market shares data from a sufficiently large number of markets $M$. Moreover, assume that product characteristics are random draws from a distribution with continuum support such that the random variables $\gamma+\mathbf{x}_{1}^{m} \beta_{0}$ cover the support of the search cost distribution $H$, where $\mathbf{x}_{1}^{m}$ is the vector of characteristics of the best product in market $m$, and $\beta_{0}$ is the true parameter vector. Then, the preference parameter vector $\beta_{0}$ and the search cost distribution $H$ are jointly identified.

The reason why pooling data from multiple markets helps identify the search cost distribution is that variation in $\mathbf{x}_{i} \beta$ across markets generates variation in the set of search cost cutoffs (22) for which the econometrician can retrieve the density of search costs. Therefore, by pooling market share data from many markets, one forces the search cost distribution to be uniquely determined for a much larger set of points.

\section{Concluding remarks}

The estimation of consumer search costs in markets constitutes a new and important area of empirical research. In this paper we have asked whether the costs of simultaneous search can be non-parametrically identified using data on the market shares and the characteristics of the different alternatives to be searched. We have shown that employing data from a single market does not suffice to identify the search cost distribution even if the utility distributions of the different alternatives are known to the econometrician.

We have then shown that by pooling market share data from multiple markets with varying alternatives and similar decision makers the search cost distribution and the utility parameters of the logit demand model are identified. We have adopted the logit demand framework for technical simplicity. However, our identification result relies on obtaining variation across markets that causes variation in the search cost cutoffs that are identified from single markets. This strategy should be successful with other demand structures.

Our study has focused on demand and search cost identification and therefore we have ignored potential endogeneity of some of the characteristics of the alternatives, for example the price. Moreover, we have assumed decision-makers are heterogeneous only in search costs. Identification of models with additional heterogeneity (mixed logit) and price endogeneity is an interesting and open avenue for further research. ${ }^{11}$

\footnotetext{
${ }^{11}$ In related work, we have studied the identification of search costs in a market with homogeneous products (cf.
} 


\section{Appendix}

Proof of Proposition 1. For notational simplicity, denote $f_{\ell} \equiv f\left(\Sigma_{\ell}\right)$ for any $\ell$. Before providing the proof we state the following auxiliary result.

Lemma 1 Let $j$ belong to $\{1,2, \ldots, h-1\}$. Under the conditions of Proposition 1 the inequality $\min \left\{\frac{f_{k+j}-f_{k}}{j}, \frac{f_{k+j}-f_{k+1}}{j-1}, \ldots, f_{k+j}-f_{k+j-1}\right\} \leq \max \left\{f_{k+j+1}-f_{k+j}, \frac{f_{k+j+2}-f_{k+j}}{2}, \ldots, \frac{f_{k+h}-f_{k+j}}{h-j}\right\}$

Proof. The inequalities in $(i i)$ can be written as

$$
\min _{\ell<k+j}\left\{\frac{f_{k+j}-f_{\ell}}{k+j-\ell}\right\} \leq \max _{\ell>k+j}\left\{\frac{f_{\ell}-f_{k+j}}{\ell-(k+j)}\right\}
$$

Note that

$$
\min _{\ell<k+j}\left\{\frac{f_{k+j}-f_{\ell}}{k+j-\ell}\right\}=\min \left\{\min _{\ell<k}\left\{\frac{f_{k+j}-f_{\ell}}{k+j-\ell}\right\}, \frac{f_{k+j}-f_{k}}{j}, \frac{f_{k+j}-f_{k+1}}{j-1}, \ldots, f_{k+j}-f_{k+j-1}\right\}
$$

and

$\max _{\ell>k+j}\left\{\frac{f_{\ell}-f_{k+j}}{\ell-(k+j)}\right\}=\max \left\{\max _{\ell>k+h}\left\{\frac{f_{\ell}-f_{k+j}}{\ell-(k+j)}\right\}, f_{k+j+1}-f_{k+j}, \frac{f_{k+j+2}-f_{k+j}}{2}, \ldots, \frac{f_{k+h}-f_{k+j}}{h-j}\right\}$.

By condition $(i)$

$$
\max _{\ell>k}\left\{\frac{f_{\ell}-f_{k}}{\ell-k}\right\} \leq \min _{\ell<k}\left\{\frac{f_{k}-f_{\ell}}{k-\ell}\right\}
$$

or

$$
\frac{f_{k+j}-f_{k}}{j} \leq \frac{f_{k}-f_{\ell}}{k-\ell} \text { for all } \ell<k
$$

Moraga-González, Sándor and Wildenbeest, 2010). The approach there is quite different because identification with endogenous prices does not require the use of instruments, as opposed to the case of differentiated products where it does. Moreover, since with homogeneous products equilibrium is characterized by mixed strategies, information on market shares is not necessary. 
which is equivalent to ${ }^{12}$

$$
\frac{f_{k+j}-f_{k}}{j} \leq \frac{f_{k+j}-f_{\ell}}{k+j-\ell} \text { for all } \ell<k,
$$

that is,

$$
\frac{f_{k+j}-f_{k}}{j} \leq \min _{\ell<k}\left\{\frac{f_{k+j}-f_{\ell}}{k+j-\ell}\right\}
$$

Therefore, by (24)

$$
\min _{\ell<k+j}\left\{\frac{f_{k+j}-f_{\ell}}{k+j-\ell}\right\}=\min \left\{\frac{f_{k+j}-f_{k}}{j}, \frac{f_{k+j}-f_{k+1}}{j-1}, \ldots, f_{k+j}-f_{k+j-1}\right\} .
$$

Further, from condition (iii)

$$
\max _{\ell>k+h}\left\{\frac{f_{\ell}-f_{k+h}}{\ell-(k+h)} \leq \min _{\ell<k+h}\left\{\frac{f_{k+h}-f_{\ell}}{k+h-\ell}\right\}\right\}
$$

or

$$
\frac{f_{\ell}-f_{k+h}}{\ell-(k+h)} \leq \frac{f_{k+h}-f_{k+j}}{h-j} \text { for all } \ell>k+h,
$$

which is equivalent to

$$
\frac{f_{\ell}-f_{k+j}}{\ell-(k+j)} \leq \frac{f_{k+h}-f_{k+j}}{h-j} \text { for all } \ell>k+h
$$

Therefore, by (25)

$$
\max _{\ell>k+j}\left\{\frac{f_{\ell}-f_{k+j}}{\ell-(k+j)}\right\}=\max \left\{f_{k+j+1}-f_{k+j}, \frac{f_{k+j+2}-f_{k+j}}{2}, \ldots, \frac{f_{k+h}-f_{k+j}}{h-j}\right\} .
$$

Based on (ii), (26), (27), we obtain the result.

We now proceed with the proof of Proposition 1. We need to prove that $\underline{c}_{k}=\frac{f_{k+h}-f_{k}}{h}$ and $\bar{c}_{k+h}=\frac{f_{k+h}-f_{k}}{h}$. Using (6) and (7), this is equivalent to showing that

$$
\begin{aligned}
& \frac{f_{k+h}-f_{k}}{h} \geq \frac{f_{\ell}-f_{k}}{\ell-k} \text { for all } k<\ell \leq N \text { and } \\
& \frac{f_{k+h}-f_{k}}{h} \leq \frac{f_{k+h}-f_{\ell}}{k+h-\ell} \text { for all } 0 \leq \ell<k+h,
\end{aligned}
$$

\footnotetext{
${ }^{12}$ This follows from

$$
\begin{aligned}
\frac{f_{k+j}-f_{k}}{j} & \leq \frac{f_{k}-f_{\ell}}{k-\ell} \text { for all } \ell<k, \\
& \mathbb{1} \\
(k-\ell)\left(f_{k+j}-f_{k}\right) & \leq j\left(f_{k}-f_{\ell}\right) \text { for all } \ell<k, \\
& \mathbb{} \\
(k-\ell)\left(f_{k+j}-f_{k}\right)+j\left(f_{k+j}-f_{k}\right) & \leq j\left(f_{k}-f_{\ell}\right)+j\left(f_{k+j}-f_{k}\right) \text { for all } \ell<k, \\
& \mathbb{}(k+j-\ell)\left(f_{k+j}-f_{k}\right) \\
& \leq j\left(f_{k+j}-f_{\ell}\right) \text { for all } \ell<k, \\
\frac{f_{k+j}-f_{k}}{j} & \leq \frac{f_{k+j}-f_{\ell}}{k+j-\ell} \text { for all } \ell<k,
\end{aligned}
$$
}


respectively. The inequality (iii) implies

$$
\begin{aligned}
& \min _{\ell<k+h}\left\{\frac{f_{k+h}-f_{\ell}}{k+h-\ell}\right\} \geq \max _{\ell>k+h}\left\{\frac{f_{\ell}-f_{k+h}}{\ell-(k+h)}\right\} \\
& \frac{f_{k+h}-f_{k}}{h} \geq \frac{f_{\ell}-f_{k+h}}{\ell-(k+h)} \text { for all } \ell>k+h,
\end{aligned}
$$

so for (28) we only need to prove that

$$
\frac{f_{k+h}-f_{k}}{h} \geq \frac{f_{k+i}-f_{k}}{i} \text { for all } i \text { with } 1 \leq i \leq h-1 .
$$

The inequality $(i)$ implies

$$
\frac{f_{k+h}-f_{k}}{h} \leq \frac{f_{k}-f_{\ell}}{k-\ell} \text { for all } \ell<k,
$$

which is equivalent to

$$
\frac{f_{k+h}-f_{k}}{h} \leq \frac{f_{k+h}-f_{\ell}}{h-\ell} \text { for all } \ell<k,
$$

so in order to show (29) we only need to prove that

$$
\frac{f_{k+h}-f_{k}}{h} \leq \frac{f_{k+h}-f_{k+i}}{h-i} \text { for all } i \text { with } 1 \leq i \leq h-1 .
$$

This is, however, equivalent to (30), so by showing (30) we can establish both (28) and (29). That is what we do in the remaining of this proof.

In proving (30) we proceed by induction with respect to $i$. First we prove (30) for $i=1$, that is,

$$
f_{k+1}-f_{k} \leq \frac{f_{k+h}-f_{k}}{h} .
$$

If $h=1$ then there is nothing to prove. Assume $h \geq 2$. By (23) for $j=1$ we have

$$
f_{k+1}-f_{k} \leq \max _{\ell \in[2, h]}\left\{\frac{f_{k+\ell}-f_{k+1}}{\ell-1}\right\}
$$

where $[2, h] \equiv\{2,3, \ldots, h\}$. Let $\ell_{1} \in[2, h]$ such that

$$
\max _{\ell \in[2, h]}\left\{\frac{f_{k+\ell}-f_{k+1}}{\ell-1}\right\}=\frac{f_{k+\ell_{1}}-f_{k+1}}{\ell_{1}-1}
$$

We argue that

$$
f_{k+1}-f_{k} \leq \min \left\{\frac{f_{k+\ell_{1}}-f_{k}}{\ell_{1}}, \frac{f_{k+\ell_{1}}-f_{k+1}}{\ell_{1}-1}, \ldots, f_{k+\ell_{1}}-f_{k+\ell_{1}-1}\right\}
$$

Indeed, (31) and (32) imply that $f_{k+1}-f_{k} \leq \frac{f_{k+\ell_{1}}-f_{k+1}}{\ell_{1}-1}$, which is equivalent to

$$
f_{k+1}-f_{k} \leq \frac{f_{k+\ell_{1}}-f_{k}}{\ell_{1}}
$$


Also, (32) implies that $\frac{f_{k+\ell}-f_{k+1}}{\ell-1} \leq \frac{f_{k+\ell_{1}}-f_{k+1}}{\ell_{1}-1}$ for any $\ell \in[2, h]$, which implies $\frac{f_{k+\ell_{1}}-f_{k+1}}{\ell_{1}-1} \leq$ $\frac{f_{k+\ell_{1}}-f_{k+\ell}}{\ell_{1}-\ell}$ for any $\ell \in\left[2, \ell_{1}-1\right]$. So $f_{k+1}-f_{k} \leq \frac{f_{k+\ell_{1}}-f_{k+\ell}}{\ell_{1}-\ell}$ for any $\ell \in\left[0, \ell_{1}-1\right]$ and (33) follows. By (23) for $j=\ell_{1}$ and (33)

$$
f_{k+1}-f_{k} \leq \max _{\ell \in\left[\ell_{1}+1, h\right]}\left\{\frac{f_{k+\ell}-f_{k+\ell_{1}}}{\ell-\ell_{1}}\right\}
$$

Let $\ell_{2} \in\left[\ell_{1}+1, h\right]$ such that

$$
\max _{\ell \in\left[\ell_{1}+1, h\right]}\left\{\frac{f_{k+\ell}-f_{k+\ell_{1}}}{\ell-\ell_{1}}\right\}=\frac{f_{k+\ell_{2}}-f_{k+\ell_{1}}}{\ell_{2}-\ell_{1}}
$$

Now, similar to (33), we argue that

$$
f_{k+1}-f_{k} \leq \min \left\{\frac{f_{k+\ell_{2}}-f_{k}}{\ell_{2}}, \frac{f_{k+\ell_{2}}-f_{k+1}}{\ell_{2}-1}, \ldots, f_{k+\ell_{2}}-f_{k+\ell_{2}-1}\right\} .
$$

Indeed, (35) and (36) imply that

$$
f_{k+1}-f_{k} \leq \frac{f_{k+\ell_{2}}-f_{k+\ell_{1}}}{\ell_{2}-\ell_{1}}
$$

which together with (33) implies $f_{k+1}-f_{k} \leq \frac{f_{k+\ell_{2}}-f_{k+\ell}}{\ell_{2}-\ell}$ for any $\ell \in\left[0, \ell_{1}\right]$. Also, (36) implies that $\frac{f_{k+\ell}-f_{k+\ell_{1}}}{\ell-\ell_{1}} \leq \frac{f_{k+\ell_{2}}-f_{k+\ell_{1}}}{\ell_{2}-\ell_{1}}$ for any $\ell \in\left[\ell_{1}+1, h\right]$, which implies $\frac{f_{k+\ell_{2}}-f_{k+\ell_{1}}}{\ell_{2}-\ell_{1}} \leq \frac{f_{k+\ell_{2}}-f_{k+\ell}}{\ell_{2}-\ell}$ for any $\ell \in\left[\ell_{1}+1, \ell_{2}-1\right]$. These imply (37).

We can continue this procedure up to some step $s$ so that we obtain

$$
f_{k+1}-f_{k} \leq \frac{f_{k+\ell_{j}}-f_{k+\ell_{j-1}}}{\ell_{j}-\ell_{j-1}} \text { for } j=1, \ldots, s,
$$

where $\ell_{0}=0$. The sequence $\left(\ell_{j}\right)_{j=1}^{s}$ is strictly increasing, so for some finite $s$ we get $\ell_{s}=h$. By summing (39) for $j=1, \ldots, s$ we get the desired result.

The next step in the induction with respect to $i$ is to assume that (30) holds for $1,2, \ldots, i-1$, that is,

$$
f_{k+1}-f_{k} \leq \frac{f_{k+h}-f_{k}}{h}, \quad \frac{f_{k+2}-f_{k}}{2} \leq \frac{f_{k+h}-f_{k}}{h}, \ldots, \quad \frac{f_{k+i-1}-f_{k}}{i-1} \leq \frac{f_{k+h}-f_{k}}{h}
$$

We now prove it for $i$. By (23) for $j=i$

$$
\min _{\ell \in[0, i-1]}\left\{\frac{f_{k+i}-f_{k+\ell}}{i-\ell}\right\} \leq \max _{\ell \in[i+1, h]}\left\{\frac{f_{k+\ell}-f_{k+i}}{\ell-i}\right\} .
$$

We treat the following two cases separately.

(i) $\min _{\ell \in[0, i-1]}\left\{\frac{f_{k+i}-f_{k+\ell}}{i-\ell}\right\}=\frac{f_{k+i}-f_{k+r}}{i-r}$ with $r \in[1, i-1]$;

(ii) $\min _{\ell \in[0, i-1]}\left\{\frac{f_{k+i}-f_{k+\ell}}{i-\ell}\right\}=\frac{f_{k+i}-f_{k}}{i}$. 
In case (i) we have

$$
\frac{f_{k+i}-f_{k+r}}{i-r} \leq \frac{f_{k+i}-f_{k}}{i}
$$

which is equivalent to

$$
\frac{f_{k+i}-f_{k}}{i} \leq \frac{f_{k+r}-f_{k}}{r},
$$

and (30) follows by (40).

In case (ii) we have

$$
\frac{f_{k+i}-f_{k}}{i} \leq \frac{f_{k+i}-f_{k+\ell}}{i-\ell} \text { for all } \ell \in[0, i-1] .
$$

Further, let $\ell_{1} \in[i+1, h]$ be such that

$$
\max _{\ell \in[i+1, h]}\left\{\frac{f_{k+\ell}-f_{k+i}}{\ell-i}\right\}=\frac{f_{k+\ell_{1}}-f_{k+i}}{\ell_{1}-i},
$$

so

$$
\frac{f_{k+i}-f_{k}}{i} \leq \frac{f_{k+\ell_{1}}-f_{k+i}}{\ell_{1}-i}
$$

by (41) and

$$
\frac{f_{k+\ell}-f_{k+i}}{\ell-i} \leq \frac{f_{k+\ell_{1}}-f_{k+i}}{\ell_{1}-i} \text { for all } \ell \in[i+1, h] .
$$

Then, similar to the proof of the case for $i=1$, we show that

$$
\frac{f_{k+i}-f_{k}}{i} \leq \min _{\ell \in\left[0, \ell_{1}-1\right]} \frac{f_{k+\ell_{1}}-f_{k+\ell}}{\ell_{1}-\ell} \text {, i.e., } \frac{f_{k+i}-f_{k}}{i} \leq \frac{f_{k+\ell_{1}}-f_{k+\ell}}{\ell_{1}-\ell} \text { for all } \ell \in\left[0, \ell_{1}-1\right] .
$$

Indeed, (42) and (43) are equivalent to

$$
\begin{aligned}
(i-\ell)\left(f_{k+i}-f_{k}\right) & \leq i\left(f_{k+i}-f_{k+\ell}\right) \text { for all } \ell \in[0, i-1] \text { and } \\
\left(\ell_{1}-i\right)\left(f_{k+i}-f_{k}\right) & \leq i\left(f_{k+\ell_{1}}-f_{k+i}\right)
\end{aligned}
$$

respectively, which by summing imply

$$
\frac{f_{k+i}-f_{k}}{i} \leq \frac{f_{k+\ell_{1}}-f_{k+\ell}}{\ell_{1}-\ell} \text { for all } \ell \in[0, i-1]
$$

Further, (44) implies

$$
\frac{f_{k+\ell_{1}}-f_{k+i}}{\ell_{1}-i} \leq \frac{f_{k+\ell_{1}}-f_{k+\ell}}{\ell_{1}-\ell} \text { for all } \ell \in\left[i+1, \ell_{1}-1\right]
$$

which by (43) implies

$$
\frac{f_{k+i}-f_{k}}{i} \leq \frac{f_{k+\ell_{1}}-f_{k+\ell}}{\ell_{1}-\ell} \text { for all } \ell \in\left[i+1, \ell_{1}-1\right]
$$


Then (45) follows by (43), (46), (47).

At this stage we can invoke the inequality (23) for $j=\ell_{1}$ to get

$$
\frac{f_{k+i}-f_{k}}{i} \leq \max _{\ell \in\left[\ell_{1}+1, h\right]}\left\{\frac{f_{k+\ell}-f_{k+\ell_{1}}}{\ell-\ell_{1}}\right\} \text {. }
$$

Let $\ell_{2} \in\left[\ell_{1}+1, h\right]$ such that

$$
\max _{\ell \in\left[\ell_{1}+1, h\right]}\left\{\frac{f_{k+\ell}-f_{k+\ell_{1}}}{\ell-\ell_{1}}\right\}=\frac{f_{k+\ell_{2}}-f_{k+\ell_{1}}}{\ell_{2}-\ell_{1}}
$$

We will show that

$$
\frac{f_{k+i}-f_{k}}{i} \leq \min _{\ell \in\left[0, \ell_{2}-1\right]}\left\{\frac{f_{k+\ell_{2}}-f_{k+\ell}}{\ell_{2}-\ell}\right\} \text {, i.e., } \frac{f_{k+i}-f_{k}}{i} \leq \frac{f_{k+\ell_{2}}-f_{k+\ell}}{\ell_{2}-\ell} \text { for all } \ell \in\left[0, \ell_{2}-1\right] .
$$

By (48) and (45)

$$
\begin{aligned}
\left(\ell_{2}-\ell_{1}\right)\left(f_{k+i}-f_{k}\right) & \leq i\left(f_{k+\ell_{2}}-f_{k+\ell_{1}}\right) \\
\left(\ell_{1}-\ell\right)\left(f_{k+i}-f_{k}\right) & \leq i\left(f_{k+\ell_{1}}-f_{k+\ell}\right) \text { for all } \ell \in\left[0, \ell_{1}-1\right]
\end{aligned}
$$

which by summation implies

$$
\frac{f_{k+i}-f_{k}}{i} \leq \frac{f_{k+\ell_{2}}-f_{k+\ell}}{\ell_{2}-\ell} \text { for all } \ell \in\left[0, \ell_{1}-1\right]
$$

By (48) and (49)

$$
\frac{f_{k+i}-f_{k}}{i} \leq \frac{f_{k+\ell_{2}}-f_{k+\ell_{1}}}{\ell_{2}-\ell_{1}}
$$

and

$$
\frac{f_{k+\ell}-f_{k+\ell_{1}}}{\ell-\ell_{1}} \leq \frac{f_{k+\ell_{2}}-f_{k+\ell_{1}}}{\ell_{2}-\ell_{1}} \text { for all } \ell \in\left[\ell_{1}+1, h\right],
$$

which implies

$$
\frac{f_{k+\ell_{2}}-f_{k+\ell_{1}}}{\ell_{2}-\ell_{1}} \leq \frac{f_{k+\ell_{2}}-f_{k+\ell}}{\ell_{2}-\ell} \text { for all } \ell \in\left[\ell_{1}+1, \ell_{2}-1\right]
$$

This together with (52) implies

$$
\frac{f_{k+i}-f_{k}}{i} \leq \frac{f_{k+\ell_{2}}-f_{k+\ell}}{\ell_{2}-\ell} \text { for all } \ell \in\left[\ell_{1}+1, \ell_{2}-1\right] .
$$

This, (51) and (52) imply (50).

Similar to the case $i=1$, we can continue this procedure up to some step $s$ so that we obtain

$$
\frac{f_{k+i}-f_{k}}{i} \leq \frac{f_{k+\ell_{j}}-f_{k+\ell_{j-1}}}{\ell_{j}-\ell_{j-1}} \text { for } j=1, \ldots, s,
$$

where $\ell_{0}=0$. The sequence $\left(\ell_{j}\right)_{j=1}^{s}$ is strictly increasing, so for some finite $s$ we get $\ell_{s}=h$. By summing (53) for $j=1, \ldots, s$ we get the desired result. 
Proof of Proposition 2. We show that $c_{k+1} \leq c_{k}$. The interesting case is when $\underline{c}_{k+1} \leq \bar{c}_{k+1}$ because if $\underline{c}_{k+1}>\bar{c}_{k+1}$ then $c_{k+1}=c_{k}$. First consider the case when $\underline{c}_{k} \leq \bar{c}_{k}$. Then by (8) and Proposition 1 (with $h=1$ ) $c_{k+1}=\underline{c}_{k+1} \leq \bar{c}_{k+1}=\underline{c}_{k}=c_{k}$. Next consider the case when $\underline{c}_{k}>\bar{c}_{k}$. Let $\ell<k$ be the largest $i$ such that $\bar{c}_{i} \geq \underline{c}_{i}$. Assume, for the moment, that $\bar{c}_{1} \geq \underline{c}_{1}$. Then $\ell \geq 1$. Then Proposition 1 implies that $\bar{c}_{k+1}=\underline{c}_{\ell}$, so $c_{k+1}=\underline{c}_{k+1} \leq \bar{c}_{k+1}=\underline{c}_{\ell}=c_{\ell}$, and since $\underline{c}_{k}>\bar{c}_{k}, \underline{c}_{k-1}>\bar{c}_{k-1}, \ldots, \underline{c}_{\ell+1}>\bar{c}_{\ell+1}$, by (8) $c_{k}=c_{k-1}=\ldots=c_{\ell}$; therefore, $c_{k+1} \leq c_{k}$. To complete the argument, we prove that $\bar{c}_{1} \geq \underline{c}_{1}$, which follows from the following Lemma because the inequality $\bar{c}_{1} \geq \underline{c}_{1}$ is equivalent to $\left(f\left(\Sigma_{\ell}\right)-f\left(\Sigma_{1}\right)\right) /(\ell-1) \leq f\left(\Sigma_{1}\right)$ for all $\ell>1$, which is equivalent to $f\left(\Sigma_{\ell}\right) / \ell \leq f\left(\Sigma_{1}\right)$ for all $\ell>1$.

Lemma $2 f\left(\Sigma_{k}\right) / k$ is decreasing in $k$ for $k \geq 1$.

Proof. We show $f\left(\Sigma_{k+1}\right) /(k+1) \leq f\left(\Sigma_{k}\right) / k$ for arbitrary $k$, which is equivalent to

$$
\int_{0}^{\bar{u}}\left(1-(k+1) \prod_{i \in \Sigma_{k}} \Psi_{i}(u)+k \prod_{i \in \Sigma_{k+1}} \Psi_{i}(u)\right) d u \geq 0 .
$$

By optimality of $\Sigma_{k}$, we know that

$$
\prod_{i \in \Sigma_{k}} \Psi_{i}(u) \leq \prod_{i \in \Sigma_{k+1} \backslash\{h\}} \Psi_{i}(u) \text { for all } h \in \Sigma_{k+1}
$$

Summing with respect to $h \in \Sigma_{k+1}$ we obtain that

$$
(k+1) \prod_{i \in \Sigma_{k}} \Psi_{i}(u) \leq \sum_{h \in \Sigma_{k+1}} \prod_{i \in \Sigma_{k+1} \backslash\{h\}} \Psi_{i}(u) .
$$

Substituting this into (54) gives

$$
\int_{0}^{\bar{u}}\left(1-(k+1) \prod_{i \in \Sigma_{k}} \Psi_{i}(u)+k \prod_{i \in \Sigma_{k+1}} \Psi_{i}(u)\right) d u \geq \int_{0}^{\bar{u}}\left(1-\sum_{h \in \Sigma_{k+1}} \prod_{i \in \Sigma_{k+1} \backslash\{h\}} \Psi_{i}(u)+k \prod_{i \in \Sigma_{k+1}} \Psi_{i}(u)\right) d u
$$

so we need to show that

$$
\int_{0}^{\bar{u}}\left(1-\sum_{h \in \Sigma_{k+1}} \prod_{i \in \Sigma_{k+1} \backslash\{h\}} \Psi_{i}(u)+k \prod_{i \in \Sigma_{k+1}} \Psi_{i}(u)\right) d u \geq 0 .
$$

This will follow from the fact that if $a_{1}, a_{2}, \ldots, a_{k+1} \in[0,1]$ and $S_{k+1}=\{1,2, \ldots, k+1\}$ then

$$
P_{k}=1-\sum_{h=1}^{k+1} \prod_{i \in S_{k+1} \backslash\{h\}} a_{i}+k \prod_{i=1}^{k+1} a_{i} \geq 0 .
$$

This can be proved by induction by noting that

$$
P_{1}=1-\left(a_{1}+a_{2}\right)+a_{1} a_{2}=\left(1-a_{1}\right)\left(1-a_{2}\right) \geq 0
$$


and

$$
P_{k}=\left(1-\prod_{i=1}^{k} a_{i}\right)\left(1-a_{k+1}\right)+a_{k+1} P_{k-1} \text { for } k \geq 2 \text {. }
$$

This is because

$$
\begin{aligned}
& \left(1-\prod_{i=1}^{k} a_{i}\right)\left(1-a_{k+1}\right)+a_{k+1} P_{k-1} \\
= & \left(1-\prod_{i=1}^{k} a_{i}\right)\left(1-a_{k+1}\right)+a_{k+1}\left(1-\sum_{h=1}^{k} \prod_{i \in S_{k} \backslash\{h\}} a_{i}+(k-1) \prod_{i=1}^{k} a_{i}\right) \\
= & 1-\prod_{i=1}^{k} a_{i}-a_{k+1}+\prod_{i=1}^{k+1} a_{i}+a_{k+1}-a_{k+1}\left(\sum_{h=1}^{k} \prod_{i \in S_{k} \backslash\{h\}} a_{i}\right)+(k-1) \prod_{i=1}^{k+1} a_{i} \\
= & 1+k \prod_{i=1}^{k+1} a_{i}-\prod_{i=1}^{k} a_{i}-a_{k+1}\left(\sum_{h=1}^{k} \prod_{i \in S_{k} \backslash\{h\}} a_{i}\right) \\
= & 1+k \prod_{i=1}^{k+1} a_{i}-\prod_{i \in S_{k+1} \backslash\{k+1\}} a_{i}-\sum_{h=1}^{k} \prod_{i \in S_{k+1} \backslash\{h\}} a_{i} \\
= & 1-\sum_{h=1}^{k+1} \prod_{i \in S_{k+1} \backslash\{h\}} a_{i}+k \prod_{i=1}^{k+1} a_{i}=P_{k} .
\end{aligned}
$$

The proof of Proposition 2 is now complete.

\section{References}

[1] Albrecht J., P. A. Gautier, and S. Vroman (2006): "Equilibrium Directed Search with Multiple Applications," Review of Economic Studies 73, 869-91.

[2] Anderson, S., and R. Renault (1999): "Pricing, Product Diversity, and Search Costs: a Bertrand-Chamberlin-Diamond model," RAND Journal of Economics, 30, 719-735.

[3] Bajari, P., J. T. Fox, K. il Kim, S. P. Ryan (2010): "The Random Coefficients Logit Model Is Identified," unpublished manuscript.

[4] Burdett, K. and K. Judd (1983): "Equilibrium Price Dispersion," Econometrica 51, 955-969.

[5] Burdett, K., S. Shi, and R. Wright (2001): "Pricing and Matching with Frictions." Journal of Political Economy 109, 1060-85.

[6] Chade, H. and L. Smith (2005): "Simultaneous Search," Mimeo, Michigan and Arizona State. 
[7] Chade, H. and L. Smith (2006): "Simultaneous Search," Econometrica 74, 1293-1307.

[8] Gale, D. and L. Shapley (1962): "College Admissions and the Stability of Marriage," American Mathematical Monthly 69, 9-15.

[9] Hong, H. and M. Shum.: "Using Price Distributions to Estimate Search Costs," RAND Journal of Economics 37, 257-75, 2006.

[10] Honka, E. (2010): "Quantifying Search and Switching Costs in the U.S. Auto Insurance Industry," unpublished manuscript.

[11] Hortaçsu, A. and C. Syverson: "Product Differentiation, Search Costs, and Competition in the Mutual Fund Industry: A Case Study of S\&P 500 Index Funds," Quarterly Journal of Economics 119, 403-56, 2004.

[12] Kim, J. B., B. J. Bronnenberg and P. Albuquerque (2009), "Online Demand under Limited Consumer Search," Marketing Science, forthcoming.

[13] Koulayev, S. (2010): "Estimating demand in online search markets, with application to hotel bookings," unpublished manuscript.

[14] Kelso, A. S., Jr., and V. P. Crawford (1982): "Job Matching, Coalition Formation, and Gross Substitutes," Econometrica 50, 1483-1504.

[15] Kircher, P. (2009): "Efficiency of Simultaneous Search," Journal of Political Economy 117, 861-913.

[16] Mehta, N., S. Rajiv, K. Srinivasan (2003): "Price Uncertainty and Consumer Search: A Structural Model of Consideration Set Formation," Marketing Science 22, 58-84.

[17] Moraga-González, J. L., Z. Sándor, and M. R. Wildenbeest (2010): "Consumer Search and Prices in the Automobile Market," unpublished manuscript.

[18] Moraga-González, J. L., Z. Sándor, and M. R. Wildenbeest (2010): "Semi-Nonparametric Estimation of Consumer Search Costs," unpublished manuscript.

[19] Roth, A. E. and M. Sotomayor (1989): "The College Admissions Problem Revisited," Econometrica 57, 559-570.

[20] Seiler, S. (2010): "The Impact of Search Costs on Consumer Behavior: A Dynamic Approach," unpublished manuscript. 
[21] Stigler, G. (1961): "The Economics of Information," Journal of Political Economy 69, 213-25.

[22] Wolinsky, A. (1986): "True Monopolistic Competition as a Result of Imperfect Information," Quarterly Journal of Economics, 101, 493-511. 